\title{
Environment effects on the electric conductivity of double stranded DNA molecules
}

\author{
A. V. Malyshev†, E. Díaz, and F Domínguez-Adame \\ GISC. Departamento de Física de Materiales, Universidad Complutense, E-28040 \\ Madrid, Spain
}

V. A. Malyshev

Zernike Institute for Advanced Materials, University of Groningen, Nijenborgh 4, 9747 AG Groningen, The Netherlands

E-mail: a.malyshev@fis.ucm.es

\begin{abstract}
.
We present a theoretical analysis of the environment effects on charge transport in double-stranded synthetic poly $(\mathrm{G})$-poly $(\mathrm{C})$ DNA molecules attached to two ideal leads. Coupling of the DNA to the environment results in two effects: (i) localization of carrier functions due to the static disorder and (ii) phonon-induced scattering of the carrier between these localized states, resulting in hopping conductivity. A nonlinear Pauli master equation for populations of localized states is used to describe the hopping transport and calculate the electric current as a function of the applied bias. We demonstrate that, although the electronic gap in the density of states shrinks as the disorder increases, the voltage gap in the $I-V$ characteristics becomes wider. Simple physical explanation of this effect is provided.

PACS numbers: 87.14.gk, 72.80.-r, 72.20.Ee
\end{abstract}

Submitted to: J. Phys.: Condens. Matter

$\ddagger$ On leave from A. F. Ioffe Physico-Technical Institute, St. Petersburg, Russia 


\section{Introduction}

Electronic transport through DNA molecules attached to leads still remains a controversial topic. A number of experiments on electrical transport through dry and wet DNA molecules revealed a variety of results. Double stranded DNA demonstrated proximity-induced superconducting [1], metallic [2-4], semiconducting [5-10] and insulating $[11,12]$ behaviors. The observed differences are often attributed to contact effects, coupling of the DNA to the environment, and to the sequence of nucleotides. Due to the diversity of experimental results, no consensus on mechanisms responsible for charge transport in the DNA has been achieved so far.

Semiconducting behavior of double stranded synthetic poly $(G)-p o l y(C)$ DNA was established experimentally. The $I-V$ characteristic of the molecule revealed a voltage gap [5]. Effective Hamiltonian models, based on the tight-binding approximation [1323], provided a reasonable description of the semiconductor gap observed in experiments with a minimum set of adjustable parameters.

Less effort has been devoted to study the effects of molecular vibrations on the electric current through DNA. It was found that hopping of the charge between the sites of guanine $(\mathrm{G})$ traps and the charge-phonon coupling results in a staircase structure of the $I-V$ characteristics [24]. The influence of vibrational modes on the electronic properties of various types of DNA molecules (synthetic and natural) was addressed in Ref. [25]. It was argued that charge transport is dominated by quasiballistic contributions in homogeneous DNA and the zero-bias conductance is enhanced by the coupling to vibrations. Dissipative effects in the electronic transport through DNA molecular wires, comprising counter-ions and hydration shells, were investigated in Ref. [26]. A bath-induced pseudo-gap opens in the strong-coupling regime and a crossover from tunneling to phonon-assisted transport was observed with increasing temperature. Also, it was claimed that disorder effects smear the electronic band but have negligible impact on the formation of the pseudo-gap.

In this work we focus on the effect of the environment on the charge transport properties of DNA molecules. To this end, we use the tight-binding ladder model [13] with random base-pair energies to describe the DNA electronic states, when disorder localizes electronic states. Charge transport is then mediated by phonon-assisted hopping between these states, which is described by means of a nonlinear Pauli master equation enabling us to calculate the current as a function of the applied voltage. We study the dependence of the $I-V$ characteristics on the magnitude of disorder.

\section{Ladder model of DNA}

We consider the ladder-like model [13] of the poly(G)-poly(c) DNA based on a tightbinding Hamiltonian in the nearest-neighbor approximation. Figure 1 represents the schematics of the model. 


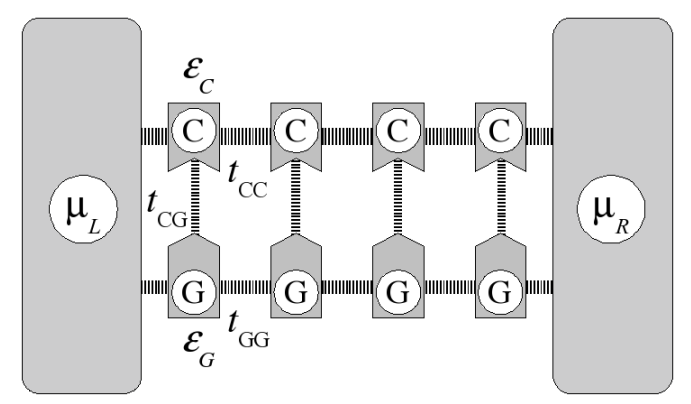

Figure 1. Schematic view of a fragment of $\operatorname{poly}(\mathrm{G})$-poly $(\mathrm{C})$ DNA molecules, excluding the sugar-phosphate backbone, coupled to ideal leads.

The corresponding Hamiltonian reads:

$$
\begin{aligned}
& \left(E-\varepsilon_{n}^{C}\right) \psi_{n}^{C}=t_{C C}\left(\psi_{n+1}^{C}+\psi_{n-1}^{C}\right)+t_{C G} \psi_{n}^{G}, \\
& \left(E-\varepsilon_{n}^{G}\right) \psi_{n}^{G}=t_{G G}\left(\psi_{n+1}^{G}+\psi_{n-1}^{G}\right)+t_{C G} \psi_{n}^{C} .
\end{aligned}
$$

Here superscripts $G$ and $C$ label a strand, $\varepsilon_{n}^{G}$ and $\varepsilon_{n}^{C}$ are energies of base molecules, $t_{G G}$ and $t_{C C}$ are intra-strand transfer interactions (from now on we use $t_{C C}=t_{G G}$ for simplicity), while $t_{C G}$ is the inter-strand interaction. In the absence of disorder, site energies are the same along each strand.

\section{Coupling to the environment}

Interactions with a random environment of solute molecules and ions surrounding the DNA molecule can (i) result in perturbation of the energies of base molecules and (ii) give rise to coupling to phonons of the bath. We account for the former effect by considering random base energies uniformly distributed within a box of width $\Delta$ and centered around the unperturbed energies $\varepsilon_{G}$ or $\varepsilon_{C}$, depending on the strand. Disorder, originated from e.g. electrostatic interactions with solute ions, can be large and all electronic states are therefore localized at segments which are typically shorter than the length of the DNA molecule. The system would therefore have exponentially small transmission coefficient and direct tunneling through the system is expected to be suppressed. On the other hand, electron-phonon coupling can result in phonon-assisted hopping between these localized states, i.e. incoherent charge transport.

For each realization of disorder, we diagonalize the Hamiltonian (1) and calculate the scattering rate between the eigenstate $\psi_{\beta}$ (with energy $E_{\beta}$ ) and another one $\psi_{\alpha}$ (with energy $E_{\alpha}$ ) according to (see Refs. [27-31] for further details):

$$
W_{\alpha \beta}=W_{0} S\left(E_{\beta}-E_{\alpha}\right) \mathcal{I}_{\alpha \beta} F\left(E_{\beta}-E_{\alpha}, T\right) .
$$

Here, the constant $W_{0}$ stands to characterize the strength of scattering. We assume a glassy host and take the spectral density function in the Ohmic form $S\left(E_{\beta}-E_{\alpha}\right)=$ $\left|E_{\beta}-E_{\alpha}\right| / t_{G G}$ widely used in the theory of dissipative systems (see, e.g. Ref. [32]). The 
temperature $T$ enters into this expression through the function $F\left(E_{\beta}-E_{\alpha}, T\right)$ defined as

$$
F\left(E_{\beta}-E_{\alpha}, T\right)=\left\{\begin{array}{ll}
1+n\left(E_{\beta}-E_{\alpha}\right), & E_{\beta}>E_{\alpha} \\
n\left(E_{\alpha}-E_{\beta}\right), & E_{\beta}<E_{\alpha}
\end{array},\right.
$$

where $n\left(E_{\beta}-E_{\alpha}\right)=\left[\exp \left(\left|E_{\beta}-E_{\alpha}\right| / T\right)-1\right]^{-1}$ is the occupation number of the vibration mode with frequency $\left|E_{\beta}-E_{\alpha}\right| / \hbar$. The term

$$
\mathcal{I}_{\alpha \beta} \equiv \sum_{s=G, C} \sum_{n=1}^{N}\left|\psi_{\alpha, n}^{s}\right|^{2}\left|\psi_{\beta, n}^{s}\right|^{2}
$$

represents the overlap integral of electronic probabilities for the states $\psi_{\alpha}$ and $\psi_{\beta}$.

We describe the process of charge transport by means of the Pauli master equation for the populations $P_{\alpha}$ of the eigenstates $\alpha$ :

$$
\dot{P}_{\alpha}=\Gamma_{\alpha}^{L}\left(f_{\alpha}^{L}-P_{\alpha}\right)+\Gamma_{\alpha}^{R}\left(f_{\alpha}^{R}-P_{\alpha}\right)+\sum_{\beta=1}^{2 N}\left[\left(1-P_{\alpha}\right) W_{\alpha \beta} P_{\beta}-\left(1-P_{\beta}\right) W_{\beta \alpha} P_{\alpha}\right]
$$

where $\alpha=1,2, \cdots, 2 N$ and $f_{\alpha}^{L, R}$ are the Fermi distribution functions for the left and right leads:

$$
f_{\alpha}^{L, R}=\left[1+\exp \left(\frac{E_{\alpha}-\mu_{L, R}}{T}\right)\right]^{-1},
$$

$\mu_{L}=E_{F}+e V$ and $\mu_{R}=E_{F}$ are the chemical potentials of the left and right leads, $E_{F}$ is the Fermi energy at equilibrium taken to be in the middle of the non-disordered DNA band gap, which is the case for Au contacts [33]. The terms $\Gamma_{\alpha}^{L}=\gamma\left(\left|\psi_{1}^{G}\right|^{2}+\left|\psi_{1}^{C}\right|^{2}\right)$ and $\Gamma_{\alpha}^{R}=\gamma\left(\left|\psi_{N}^{G}\right|^{2}+\left|\psi_{N}^{C}\right|^{2}\right)$ measure the coupling between leads and the eigenstate $\alpha$, with the parameter $\gamma$ being the strength of the coupling.

We are interested in the steady state solution of Eq. (5). Solving the corresponding system of nonlinear algebraic equations by an iterative method that guarantees the condition $0 \leq P_{\alpha} \leq 1$, we obtain the stationary current as:

$$
I(V)=\sum_{\alpha=1}^{2 N} \Gamma_{\alpha}^{R}\left(f_{\alpha}^{R}-P_{\alpha}\right)
$$

\section{Results}

In all calculations, we considered 30 base-pair poly $(\mathrm{G})$-poly $(\mathrm{C})$ DNA molecules and used the following model parameters: unperturbed site energies $\varepsilon_{n}^{G}=1.14 \mathrm{eV}$ and $\varepsilon_{n}^{C}=-1.06 \mathrm{eV}$ [34], while hopping integrals were adjusted [22] to reproduce the currentvoltage characteristics measured in experiments on dry poly $(\mathrm{G})$-poly $(\mathrm{C})$ DNA [5]: $t_{G G}=t_{C C}=0.27 \mathrm{eV}$ and $t_{C G}=0.25 \mathrm{eV}$. These values are within reasonable parameter intervals [35]. We assumed that the temperature is slightly below the freezing point of the environment $(T=273 \mathrm{~K})$ which allowed us to neglect all effect related to dynamic disorder (time-dependent fluctuations of the configuration of solute ions surrounding the molecule) and validates the model, namely we consider only interaction with the phonons 

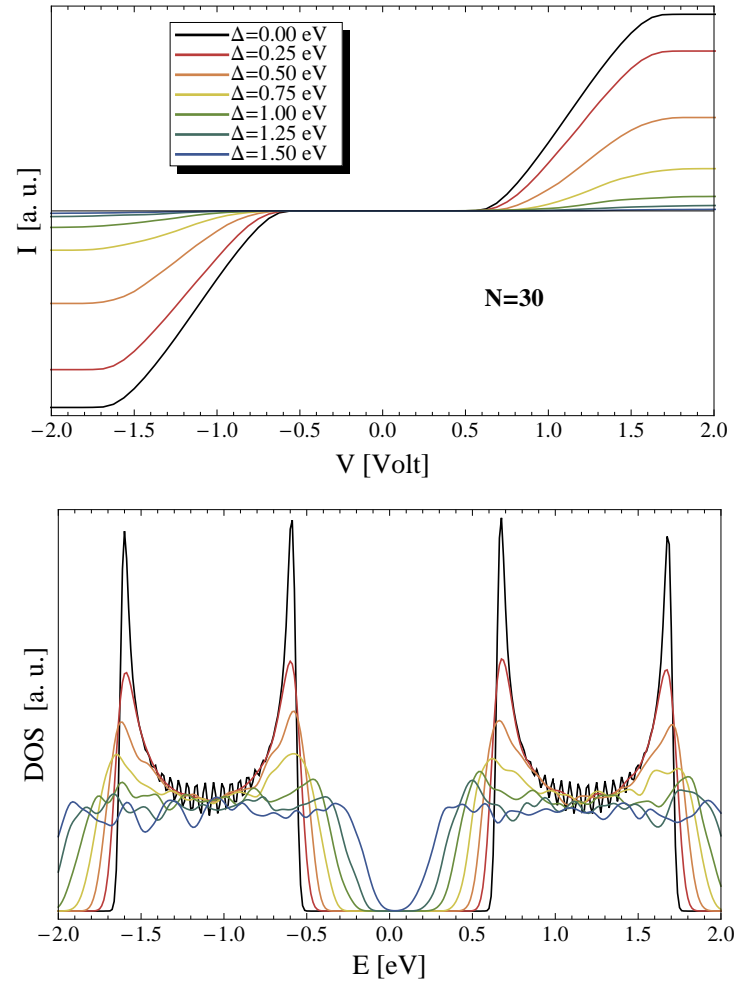

Figure 2. Current-voltage characteristics obtained for different magnitudes of disorder indicated in the legend (upper panel) and the corresponding density of states (lower panel).

of the thermal bath. The parameter $\gamma$ was found to only influence the amplitude of the current and was taken to be $\gamma=W_{0}$ in all calculations.

In the upper panel of Fig. 2 we present the calculated current-voltage characteristics obtained for different magnitudes of disorder $\Delta=0 \div 1.5 \mathrm{eV}$ (indicated in the legend). The lower panel shows the corresponding density of states. These results were averaged over 100 realizations of disorder for each value of $\Delta$. The figure demonstrates that the electronic gap in the density of states is shrinking upon increasing the disorder while the voltage gap becomes wider, which could seem counterintuitive. The closure of the electronic gap is the usual consequence of disorder. It is due to the appearance of disorder-induced states in the bare band gap, which form the tails of the density of states. It may seem that these states can give rise to current at smaller voltage drops and to the consequent shrinking of the voltage gap. Fig. 2 shows just the opposite dependence of the voltage gap, namely it broadens with increasing disorder.

In order to understand this counterintuitive dependence of the voltage gap, the following reasoning is in place. Although there are states in the energy gap (i.e. between bare band edges: $|E| \leq 0.5 \mathrm{eV}$ in Fig. 2), these states are strongly localized, so that direct tunneling via them is suppressed. In this case, only phonon-assisted hopping can give rise to charge transport. If two states are localized at different segments of the DNA, their wavefunctions have exponentially small overlap $\mathcal{I}_{\alpha \beta}$ [see Eq. (4)], and 
therefore, the scattering rate $W_{\alpha \beta}$ between the two states, given by Eq. (2), is small. On the other hand, for two well-overlapped states, the typical energy spacing is large due to the quantum mechanical level repulsion [36]. Such level spacing grows on increasing the disorder strength, reducing the thermally activated conductivity.

The typical energy separation $\delta E$ between well-overlapped states can be estimated using the argumentation described in Refs. [37-39], where it was applied to the energy range close to the band-edge. Here, we use a similar reasoning for the band center. Briefly, a state extended over a segment of size $N^{*}$ senses not the bare on-site disorder, but rather a reduced one (averaged over the localization length) with a typical magnitude of $\sigma / \sqrt{N^{*}}$, were $\sigma$ is the standard deviation of the on-site disorder distribution $(\Delta / \sqrt{12}$ in our case). The energy separation $\delta E$ is a function of $N^{*}$ (the latter magnitude is considered to be large: $\left.N^{*} \gg 1\right)$. Then the equality $\delta E\left(N^{*}\right)=\Delta / \sqrt{12 N^{*}}$ provides a self-consistent estimate for $\delta E\left(N^{*}\right)$ (see Refs. [37-39] for more details). Applying this equality to states in the center of the band associated with the $\mathrm{G}$ strand and taking into account that, for these states, $\delta E\left(N^{*}\right) \approx 2 \pi\left|t_{\mathrm{GG}}\right| / N^{*}$ we obtain

$$
\delta E \approx \frac{1}{24 \pi}\left|t_{\mathrm{GG}}\right|\left|\frac{\Delta}{t_{\mathrm{GG}}}\right|^{2} .
$$

For the typical considered disorder, $\Delta \approx 1 \mathrm{eV}$ the above estimate gives $\delta E \approx 50 \mathrm{meV}$, the energy being larger than the considered temperature $(\approx 25 \mathrm{meV})$. The latter means that the phonon occupation number in (3) is small: $n\left(\left|E_{\beta}-E_{\alpha}\right| / T\right) \ll 1$, and suggests that phonon-assisted scattering from lower to higher states is suppressed and almost no temperature activated transport can take place in the system; hops from higher to lower well-overlapped states constitute therefore the dominant scattering process. Then, the only way for a charge carrier to hop from one lead to another is to make a series of cascade-like down hops over well-overlapped states. Appropriate sets of such states can only be found in the energy region above the bare energy band edge [31,40]. Disorder smears out the band edge, pushing the boundary of this region of "conducting" states up towards the band center (this boundary is analogous to the diffusion mobility edge [40]). Thus, for a larger value of disorder, such diffusion mobility edge lies at a higher energy and, therefore, a greater voltage is required to induce electric current through the system. The latter explains the observed dependence of the voltage gap on the disorder magnitude.

The number of cascade states is of the order of $N / N^{*}$, where $N^{*}$ stands for the localization length in the appropriate part of the spectrum. Because consecutive cascade states should overlap well, they are separated by the energy of the order of $\delta E$ given by Eq. (8). Then the smallest difference between the highest and the lowest energy levels in a cascade-like set of states can be estimated as

$$
\Delta E_{\min } \approx \frac{N}{N^{*}} \delta E \approx \frac{N}{18(4 \pi)^{3}}\left|t_{\mathrm{GG}}\right|\left|\frac{\Delta}{t_{\mathrm{GG}}}\right|^{4} .
$$

In deriving of Eq. (9) we have used $\delta E \approx \Delta / \sqrt{12 N^{*}}$ (see the preceding paragraph for details). Bearing in mind that the lowest state in a cascade should be about the bare 
band edge $E_{0} \approx 0.5 \mathrm{eV}$, we conclude that the quantity $E_{0}+\Delta E_{\min }$ shows the dependence of the voltage gap on both the disorder and the system size. The former supports the above argument on the increase of the gap with disorder while the latter suggests that the voltage gap increases with the system size.

Note that the latter trend could be reversed for very short DNA chains whose length is of the order of the smallest typical localization length. In this situation all states are coupled to both leads and contribute to charge transport. Thus, the current appear as soon as the Fermi level of a contact aligns with the lowest state in the energy gap and therefore the voltage gap coincides with the energy gap and decreases with disorder (see Fig. 2). However, in experiments the chain length is typically much larger that the charge carrier localization length, $N \gg N^{*}$, so the dependence given by Eq. (9) should hold, which suggests, in particular, that long DNA chains are insulating.

\section{Conclusions}

We considered theoretically the charge transport through a synthetic double-stranded poly $(\mathrm{G})-\operatorname{poly}(\mathrm{C})$ DNA molecule attached to two ideal leads and embedded into a random static environment (e.g. solvent below its freezing temperature). A nonlinear Pauli master equation for the populations of localized electronic states was used to describe the hopping transport of charge carriers. We demonstrated that the voltage gap becomes wider as disorder increases. The calculated $I-V$ curves could indicate that the conductivity is band-like, although the charge transport is incoherent. This suggests that care should be taken when concluding on the nature of the charge transport by examining only $I-V$ characteristics. The proposed method of electric current calculation is applicable for a broader range of systems, e.g., organic polymers.

\section{Acknowledgments}

This work was supported by Ramón y Cajal Program, MEC (Project MOSAICO), and BSCH-UCM (Project PR34/07-15916).

\section{References}

[1] A. Yu. Kasumov, M. Kociak, S. Guéron, B. Reulet, V. T. Volkov, D. V. Klinov, and H. Bouchiat, Science 291, 280 (2001).

[2] Y. Okahata, T. Kobayashi, K. Tanaka, and M. J. Shimomura, J. Am. Chem. Soc. 120, 6165 (1998).

[3] H. W. Fink and C. Schönenberger, Nature 398, 407 (1999).

[4] A. Rakitin, P. Aich, C. Papadopoulos, Yu. Kobzar, A. S. Vedeneev, J. S. Lee, and J. M. Xu, Phys. Rev. Lett. 86, 3670 (2001).

[5] D. Porath, A. Bezryadin, S. de Vries, and C. Dekker, Nature 403, 635 (2000).

[6] K.-H. Yoo, D. H. Ha, J.-O. Lee, J. W. Park, J. Kim, J. J. Kim, H.-Y. Lee, T. Kawai, and H. Y. Choi, Phys. Rev. Lett. 87, 198102 (2001).

[7] J. S. Hwang, K. J. Kong, D. Ahn, G. S. Lee, D. J. Ahn, and S. W. Hwang, Appl. Phys. Lett. 81, $1134(2002)$. 
[8] B. Q. Xu, P. M. Zhang, X. L. Li, and N. J. Tao, Nano Lett. 4, 1105 (2004).

[9] H. Cohen, C. Nogues, R. Naaman, and D. Porath, Proc. Natl. Acad. Sci. 102, 11589 (2005).

[10] S. Roy, H. Vedala, A. Datta Roy, D.-H. Kim, M. Doud, K. Mathee, H.-K. Shin, N. Shimamoto, V. Prasad, and W. Choi, Nano Lett. 8, 26 (2008).

[11] E. Braun, Y. Eichen, U. Sivan, and G. Ben-Yoseph, Nature 391, 775 (1998).

[12] A. J. Storm, J. van Noort, S. de Vries, and C. Dekker, Appl. Phys. Lett. 79, 3881 (2001).

[13] K. Iguchi, Int. J. Mod. Phys. B 11, 2405 (1997).

[14] K. Iguchi, J. Phys. Soc. Jpn. 70, 593 (2001).

[15] G. Cuniberti, L. Craco, D. Porath, and C. Dekker, Phys. Rev. B 65, 241314 (2002).

[16] S. Roche, D. Bicout, E. Maciá, and E. Kats, Phys. Rev. Lett. 91, 228101 (2003).

[17] K. Iguchi, Int. J. Mod. Phys. 13, 1845 (2004).

[18] H. Yamada, Phys. Lett. A 332, 65 (2004); Int. J. Mod. Phys. B 18, 1697 (2004); Phys. Rev. B 69, 014205 (2004).

[19] V. M. Apalkov and T. Chakraborty, Phys. Rev. B 71, 033102 (2005).

[20] D. Klotsa, R. A. Römer, and M. S. Turner, Biophys. J. 89, 2187 (2005).

[21] A. Rodríguez, R. A. Römer, and M. S. Turner, phy. stat. solidi (b) 243, 373 (2006).

[22] A. V. Malyshev, Phys. Rev. Lett. 98, 096801 (2007).

[23] E. Díaz, A. V. Malyshev, y F. Domínguez-Adame, Phys. Rev. B 76, 205117 (2007).

[24] V. Apalkov and T. Chakraborty, Phys. Rev. B 72, 161102 (2005).

[25] B. B. Schmidt, M. H. Hettler, and G. Schön, Phys. Rev. B 75, 115125 (2007).

[26] R. Gutiérrez, S. Mandal, and G. Cuniberti, Phys. Rev. B 71, 235116 (2005).

[27] J. A. Leegwater, J. R. Durrant, and D. R. Glug, J. Phys. Chem. B 101, 7205 (1977);

[28] M. Bednarz, V. A. Malyshev, J. P. Lemaistre, and J. Knoester, J. Lumin. 94-95, 271 (2001).

[29] M. Shimizu, S. Suto, and T. Goto, J. Chem. Phys. 114, 2775 (2001).

[30] M. Bednarz, V. A. Malyshev, and J. Knoester, J. Chem. Phys. 120, 3827 (2004).

[31] A. V. Malyshev, V. A. Malyshev, and F. Domínguez-Adame, Chem. Phys. Lett. 371, 417 (2003); J. Phys. Chem. B 107, 4418 (2003).

[32] U. Weiss, Quantum Dissipative Systems; World Scientific: Singapore, 1993.

[33] M. S. Xu, S. Tsukamoto, S. Ishida, M. Kitamura, Y. Arakawa, R. G. Endres, and M. Shimoda, Appl. Phys. Lett. 87, 083902 (2005).

[34] H. Mehrez and M. P. Anantram, Phys. Rev. B 71, 115405 (2005).

[35] E. Maciá and S. Roche, Nanotechnology 17, 3002 (2006).

[36] A. V. Malyshev, V. A. Malyshev, and J. Knoester, Phys. Rev. Lett. 98, 087401 (2007).

[37] V. A. Malyshev, Opt. Spektrosk. 71, 873 (1991) [Opt. Spectrosc. 71, 505 (1991)]; J. Lumin. 55, 225 (1993).

[38] V. Malyshev and P. Moreno, Phys. Rev. B 51, 14587 (1995).

[39] A. V. Malyshev and V. A. Malyshev, Phys. Rev. B 63, 195111 (2001); J. Lumin. 94, 369 (2001).

[40] A. V. Malyshev, phys. stat. sol. (c) 3, 3539 (2006). 